\title{
LncRNA NKILA was upregulated in diabetic cardiomyopathy with early prediction values
}

\author{
QINGYONG LI ${ }^{1}$, PANRONG LI ${ }^{1}$, JINLING SU ${ }^{1}$, SUPING LIU ${ }^{1}$, \\ XIAOMAN YANG ${ }^{1}$, YUEJIN YANG ${ }^{2}$ and SUOCHENG NIU ${ }^{1}$ \\ ${ }^{1}$ The Second Department of Cardiology, The People's Hospital of Puyang, Puyang, Henan 457000; \\ ${ }^{2}$ Fuwai Cardiovascular Hospital, Chinese Academy of Medical Sciences, Beijing 100037, P.R. China
}

Received October 20, 2018; Accepted May 3, 2019

DOI: $10.3892 /$ etm.2019.7671

\begin{abstract}
Nuclear factor- $\kappa \mathrm{B}$ interacting long non-coding RNA (LncRNA NKILA) is a well-studied tumor suppressor lncRNA in several types of malignancies. The present study reports the involvement of this IncRNA in diabetic cardiomyopathy (DC). A 8-year-follow-up study on 312 diabetic patients without exhibiting obvious complications demonstrated that plasma lncRNA NKILA levels were upregulated specifically in diabetic patients who developed DC but not in patients with other complications. Plasma levels of lncRNA NKILA at 6 months prior to diagnosis is sufficient to distinguish patients with DC from other diabetic patients without significant complications. Although in vitro experiments demonstrated that IncRNA NKILA expression in cardiomyocyte cells was not affected by high-glucose treatment, ectopic 1ncRNA NKILA expression and lncRNA NKILA knockdown potentiated, and inhibited cardiomyocyte apoptosis, respectively. Therefore, the data from the present study suggests that overexpression of IncRNA NKILA is involved in DC, and overexpression of lncRNA NKILA may serve as a therapeutic target for treating DC.
\end{abstract}

\section{Introduction}

The development and progression of diabetes results in a series of complications that affect most major organs in the human body (1). Diabetic complications are now considered as one of the leading causes of mortality in many countries (2,3). Chronic hyperglycemia in diabetic patients promotes the expression of pyruvate dehydrogenase kinase-4, which inhibits the activity of pyruvate decarboxylase, and inhibits the expression of glucose transporters (4). The metabolic remodeling damages the

Correspondence to: Dr Suocheng Niu, The Second Department of Cardiology, The People's Hospital of Puyang, No. 252 Shengli Middle Road, Puyang, Henan 457000, P.R. China

E-mail: schniu@163.com

Key words: diabetic cardiomyopathy, nuclear factor- $\kappa \mathrm{B}$ interacting long non-coding RNA, cardiomyocyte myocardium and leads to the development of DC (5). Diabetic cardiomyopathy (DC) is ventricular dysfunction developed in diabetic patients that is not caused by hypertension or coronary artery disease (6). DC affects a considerable portion of diabetic patients, and its incidence is increasing as the disease progresses (7). DC is associated with high mortality rates even with active treatment (8). Therefore, at present, prevention of DC, rather than treatment, is more critical for the survival of diabetic patients.

A growing body of literature has shown that non-coding RNAs (ncRNAs) are key players in vascular complications associated with diabetes (9). Long non-coding RNAs (lncRNAs) are a subgroup of ncRNAs that are of $>200$ nucleotides in length (10). At present, several lncRNAs have been proven to be key players in DC, and their the regulation may contribute to the control of disease conditions $(11,12)$. Nuclear factor- $\kappa \mathrm{B}$ interacting long non-coding RNA (LncRNA NKILA) is a well-studied tumor suppressor IncRNA in several types of malignancies $(13,14)$, while its involvement in other human diseases is unknown. The present study aimed to explore the role of lncRNA NKILA $p$ in DC.

\section{Materials and methods}

Subjects and specimens. A total of 312 diabetic patients without obvious complications that were admitted to The People's Hospital of Puyang (Henan, China) between January 2008 and January 2010 were included in the present study. The inclusion criteria were as follows: i) Patients without obvious complications in major organs; ii) patients with complete medical records; and iii) patients completed a 8-year-follow-up period. The exclusion criteria were as follows: i) Patients suffering from complications of other severe diseases, including heart disease; ii) patients failed to cooperate with researchers; and iii) patients succumbed during follow-up period. These patients consisted of 168 males and 144 females, between 31 and 62 years of age (mean, 45.8 \pm 5.7 ). All patients signed informed consent forms before admission. The present study was approved by the Ethics Committee of The People's Hospital of Puyang.

Follow-up. All patients were followed up over a period of 8 years to record the occurrence of diabetic complications. A volume of blood $(5 \mathrm{ml})$ was extracted every 6 months to 
obtain plasma samples, which were in tern obtained via centrifugation at room temperature in EDTA tubes for $14 \mathrm{~min}$ at $1,200 \times \mathrm{g}$. Diagnostic criteria of DC were as follows: i) Diagnosed diabetes; ii) clinical manifestations of heart failure; iii) heart enlargement with impaired cardiac systolic function or diastolic dysfunction in cases of no heart enlargement; and iv) heart failure caused by other heart diseases including hypertensive heart disease. Patients with coronary heart disease and rheumatic valvular heart disease were excluded from the present study.

Reverse transcription-quantitative polymerase chain reaction $(R T-q P C R)$. To measure the expression of lncRNA NKILA, total RNA was extracted from the plasma (obtained from patients) or primary human cardiomyocyte cells using TRIzol (Invitrogen; Thermo Fisher Scientific, Inc.), according to manufacturer's protocols. Reverse transcription was performed using Applied Biosystems ${ }^{\mathrm{TM}}$ High-Capacity cDNA Reverse Transcription Kit (Applied Biosystems; Thermo Fisher Scientific, Inc.) using the following thermocycling conditions: $25^{\circ} \mathrm{C}$ for $5 \mathrm{~min}, 52^{\circ} \mathrm{C}$ for $15 \mathrm{~min}$ and $85^{\circ} \mathrm{C}$ for $5 \mathrm{~min}$. The subsequent qPCR reactions were prepared using SYBR ${ }^{\circledR}$ Green Quantitative RT-qPCR Kit (Sigma-Aldrich; Merck KGaA). The primers of lncRNA NKILA and $\beta$-actin were designed and synthesized by GenePharma (Shanghai GenePharma Co., Ltd.): NKILA forward, 5'-AACCAAACC TACCCACAACG-3' and reverse, 5'-ACCACTAAGTCAATC CCAGGTG-3'; $\beta$-actin forward, 5'-GCACCACACCTTCTA CAAT-3' and reverse, 5'-TGCTTGCTGATCCACATCTG-3'. The PCR reactions were carried out using the following thermocycling conditions: $1 \mathrm{~min}$ at $95^{\circ} \mathrm{C}, 15 \mathrm{sec}$ at $95^{\circ} \mathrm{C}$ and $30 \mathrm{sec}$ at $55.5^{\circ} \mathrm{C}$ for a total of 40 cycles. The expression of lncRNA NKILA was normalized to the endogenous control $\beta$-actin using the $2^{-\triangle \Delta C q}$ method (15).

Cells and cell transfection. Primary human cardiomyocyte cells (T4037; Applied Biological Materials) were cultured in this study to perform in vitro cell experiments under conditions as recommend by the manufacturer. Cells were cultivated in cardiomyocyte growth medium (ScienCell Research Laboratories, Inc.) at $37^{\circ} \mathrm{C}$ with $95 \%$ humidity and $5 \% \mathrm{CO}_{2}$. Vectors (pcDNA3) expressing lncRNA NKILA and its corresponding empty vector, as well as the lncRNA NKILA siRNA (5'-AUCUGGGGUAGGCGCUGGGUAU-3') with its respective negative control, were all designed and prepared by Sangon (Sangon Biotech Co., Ltd.). Lipofectamine ${ }^{\circledR} 2000$ reagent (11668-019.; Invitrogen; Thermo Fisher Scientific, Inc.) was used for all cell transfections of vectors $(10 \mathrm{nM})$ and siRNAs $(30 \mathrm{nM})$. All experiments were performed in accordance with the manufacturer's instructions. Transfection with empty vectors or negative control siRNAs was considered as the negative control (NC) whereas cells that were treated with only Lipofectamine 2000 reagent without vectors or siRNAs were considered control (C) cells. Incubation with the transfection mixture was performed for $5 \mathrm{~h}$ at $37^{\circ} \mathrm{C}$. The interval between transfection and subsequent experiments was $24 \mathrm{~h}$.

Cell apoptosis assay. IncRNA NKILA expression reached $200 \%$ and knockdown rate reached $50 \%$ at $24 \mathrm{~h}$ after the transfection of siRNA and vectors. Following $24 \mathrm{~h}$ transfection, suspensions of primary human cardiomyocyte cells at a density of $5 \times 10^{4}$ cells $/ \mathrm{ml}$ were prepared using serum-free cell culture medium (as aforementioned) supplemented with $20 \mathrm{mM}$ D-glucose, before being subsequently seeded into 6-well plates at $2 \mathrm{ml} /$ well. The cells were then cultured for $48 \mathrm{~h}$, followed by digestion using $0.25 \%$ trypsin (Sangon Biotech Co., Ltd.) before being subjected to Annexin V-Fluorescein isothiocyanate (FITC; Dojindo Molecular Technologies, Inc.) and propidium iodide (PI; Dojindo Molecular Technologies, Inc.) staining. Apoptotic cells were detected using a flow cytometer. Data were analysed using FCS Express 6 Flow Cytometry Software (De Novo Software).

Statistical analysis. Experiments were repeated three times and data were expressed as mean \pm standard deviation. All statistical analyses were performed using the SPSS19.0 software (IBM Corp.). Comparisons between two groups were performed by Student's t-test, whereas comparisons between three groups were performed using one-way analysis of variance followed by Tukey test. Diagnostic values of plasma lncRNA NKILA for DC were evaluated by applying receiver operating characteristic (ROC) curve analysis (Graphpad prism 6; GraphPad, Inc.), with DC patients as true positive cases, and diabetic patients without obvious complications as true negative cases. $\mathrm{P}<0.05$ was considered to indicate a statistically significant difference.

\section{Results}

LncRNA NKILA is upregulated in diabetic patients with DC but not in patients with other complications. At the end of the 8-year follow-up period, 48 patients were diagnosed with DC only (DC), 42 patients were diagnosed with diabetic nephropathy only (DN), 34 patients were diagnosed with diabetic retinopathy only (DR); while no significantly complications were observed in 44 diabetic patients (D), and 144 patients were diagnosed with multiple complications. The group containing 144 patients with multiple complications were excluded in the following analyses to avoid ambiguity. The expression of lncRNA NKILA in plasma collected from the different patient groups at the end of follow-up was analyzed using RT-qPCR. LncRNA NKILA was demonstrated to be significantly upregulated in the group of diabetic patients who developed DC compared with all the remaining patient groups (Fig. 1).

Plasma lncRNA NKILA levels at 6 months before diagnosis in patients with DC and diabetic patients without obvious complications. Diagnostic values of plasma lncRNA NKILA were evaluated using ROC curve analysis, with DC patients as true positive cases, and diabetic patients without any obvious complications as true negative cases. For plasma levels of lncRNA NKILA at 6 months before diagnosis, the area under the curve was 0.83 , with a standard error of 0.041 and $95 \%$ confidence interval of 0.75-0.91 (Fig. 2). However, plasma levels of lncRNA NKILA before this time point failed to diagnose DC (data not shown).

Nevertheless, ROC curve analysis carried out in the present study illustrated that plasma lncRNA NKILA levels at 6 months before diagnosis is sufficient to distinguish DC patients from diabetic patients without obvious complications. 


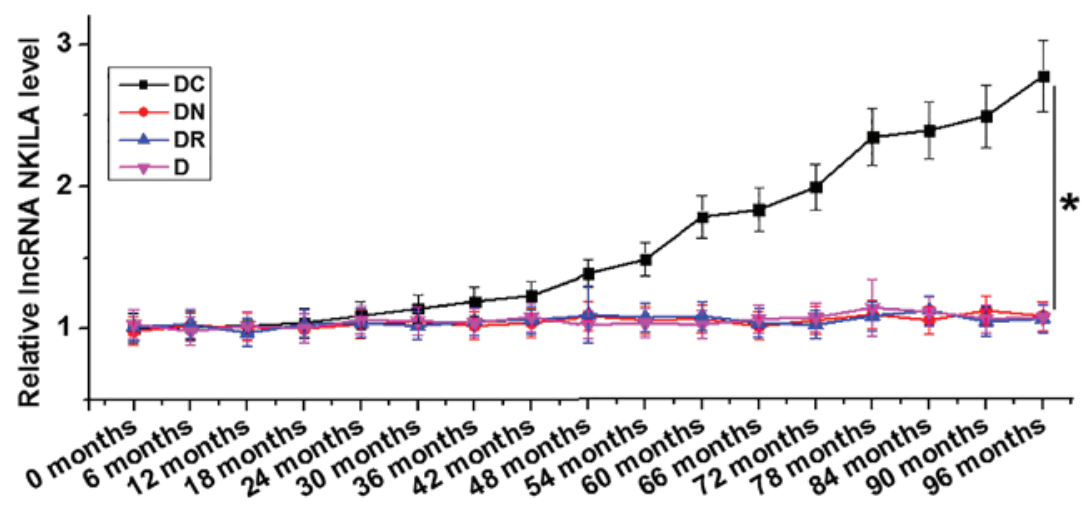

Figure 1. LncRNA NKILA is upregulated specifically in diabetic patients with DC but not in patients with other complications " $\mathrm{P}<0.05$ vs. the D group. DC, patients with DC only; DN, patients with diabetic nephropathy only; DR, patients with diabetic retinopathy only; D, patients with diabetes with no significant complications; IncRNA NKILA, nuclear factor-кB interacting long non-coding RNA.

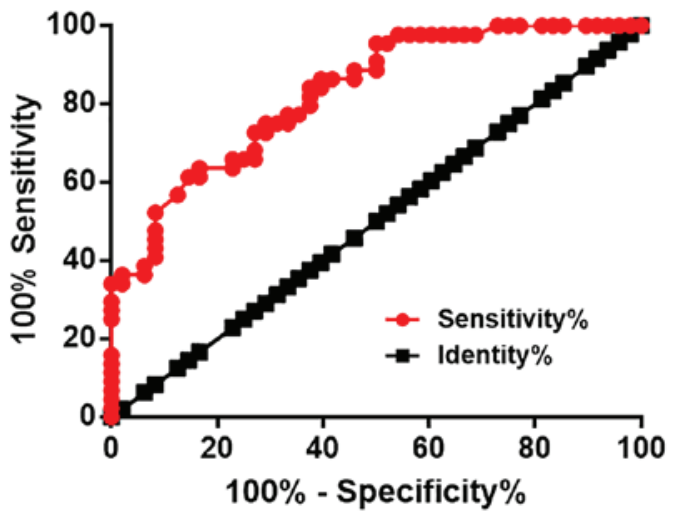

Figure 2. Plasma levels of nuclear factor $\kappa \mathrm{B}$-long non-coding RNA at 6 months before diagnosis distinguishes DC patients from diabetic patients without obvious complications (as evaluated by receiver operating characteristic curve analysis).

Expression of IncRNA NKILA in cardiomyocytes is not affected by high-glucose treatment. To investigate the effect of glucose on lncRNA NKILA expression, primary cultured human cardiomyocytes were treated with D-glucose at concentrations of 5, 10 and $20 \mathrm{mM} \mathrm{(5} \mathrm{mM} \mathrm{was} \mathrm{used} \mathrm{as} \mathrm{control} \mathrm{as} \mathrm{it}$ is within the normal blood glucose range) for 12, 24 and $36 \mathrm{~h}$. Expression of lncRNA NKILA in cardiomyocytes following D-glucose treatment was measured using RT-qPCR. Treatment with D-glucose at concentrations of 5 (control), 10 and $20 \mathrm{mM}$ for 12, 24 and $36 \mathrm{~h}$ did not lead to any significant effects on lncRNA NKILA expression in cardiomyocytes (Fig. 3).

LncRNA NKILA overexpression promotes apoptosis in cardiomyocytes. Apoptosis of cardiomyocytes contributes to the pathogenesis of DC (6). Therefore, the effects of IncRNA NKILA overexpression and depletion by siRNA knockdown on cardiomyocyte apoptosis was analyzed using Annexin V-FITC/PI staining by flow cytometry following $20 \mathrm{mM}$ D-glucose treatment. Following $24 \mathrm{~h}$ transfection, ectopic expression of the lncRNA NKILA vector in cardiomyocytes resulted in a two-fold increase observed in intracellular lncRNA NKILA mRNA levels (Fig. 4A). IncRNA NKILA siRNA transfection induced a $50 \%$ reduction in intracellular lncRNA NKILA mRNA levels compared

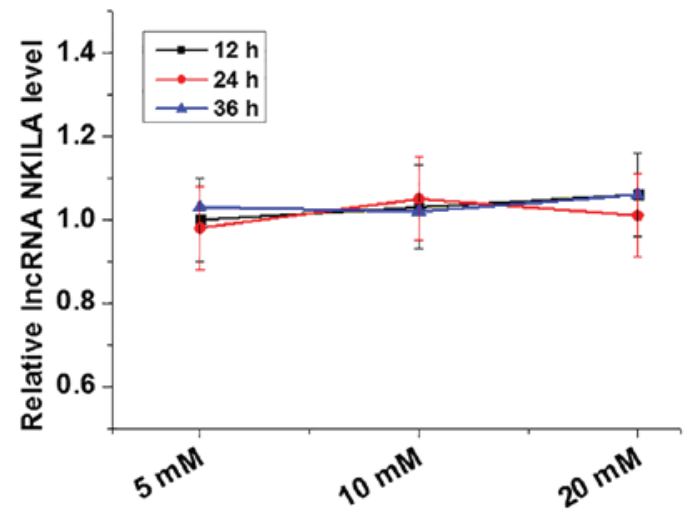

Figure 3. Expression of 1ncRNA NKILA in cardiomyocyte cells is not affected by high-glucose treatment. LncRNA NKILA, nuclear factor- $\kappa \mathrm{B}$ interacting long non-coding RNA.

with controls (Fig. 4A). These efficiencies suggest that the transfection was successful (Fig. 4A). Compared with $\mathrm{C}$ and NC groups, ectopic lncRNA NKILA expression and lncRNA NKILA knockdown accelerated and inhibited cardiomyocyte apoptosis, respectively (Fig. 4B).

\section{Discussion}

LncRNA NKILA serves a role as tumor suppressor in several types of malignancies $(13,14)$. To the best of our knowledge, the involvement of IncRNA NKILA in diabetic complications remains poorly understood. The key finding of the present study is that lncRNA NKILA was upregulated in diabetic patients who developed DC but not in patients with other complications. The experimental data presented here demonstrated that lncRNA NKILA promotes apoptosis in cardiomyocytes, which may contribute to the progression of DC.

Expression of IncRNA NKILA has been extensively investigated in many types of cancer in humans $(13,14,16,17)$. As a tumor suppressor lncRNA, NKILA expression has been illustrated to be downregulated in various types of cancer malignancies, while its upregulation inhibits cancer development (16). The 8-year-follow-up study presented here revealed that IncRNA NKILA expression was specifically upregulated with the occurrence of DC. In addition, 

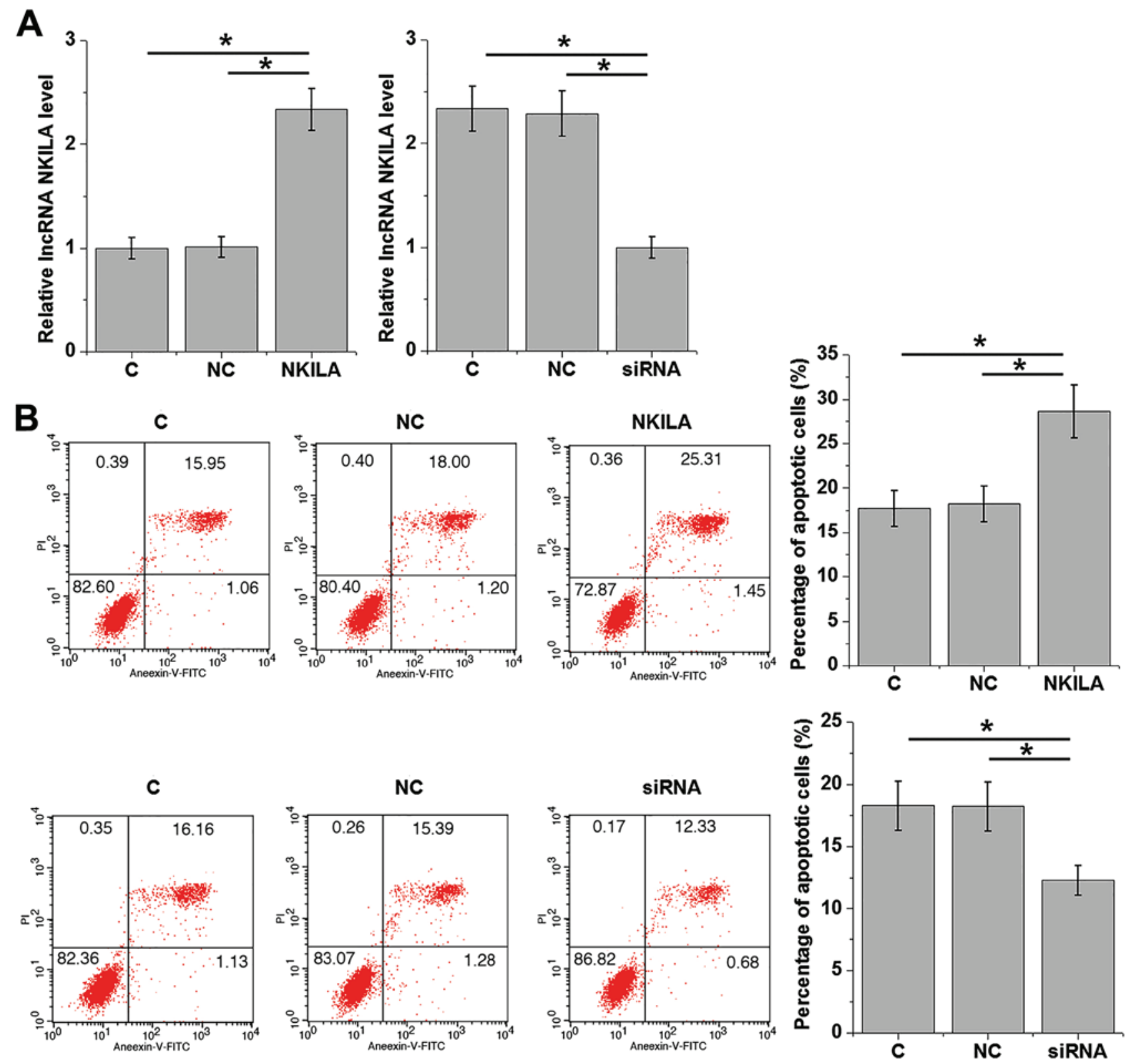

Figure 4. LncRNA NKILA promotes apoptosis of cardiomyocytes. (A) Ectopic expression of the lncRNA NKILA vector doubled the levels of lncRNA NKILA mRNA and knockdown rate reached $50 \%$ at $24 \mathrm{~h}$ after transfection. (B) LncRNA NKILA overexpression accelerated, while lncRNA NKILA knockdown inhibited the apoptosis of cardiomyocytes, respectively. "P<0.05 vs. C and NC. LncRNA NKILA, nuclear factor-kB interacting long non-coding RNA; $\mathrm{C}$, control; NC, negative control; siRNA, small interfering RNA.

lncRNA NKILA expression in cardiomyocytes was not significantly affected by high-glucose treatment, indicating that the involvement of IncRNA NKILA is specific to DC and not in diabetes per se or other complications associated with diabetes.

The mortality rate of patients with DC is unacceptably high even after active treatment (18). Therefore, development of early prediction markers for DC is critical for developing prevention and management strategies. In the present study the measurement of plasma lncRNA NKILA mRNA levels at 6 months before diagnosis was sufficient to distinguish patients with DC from patients with diabetes but without significant complications. Therefore, plasma levels of lncRNA NKILA may act as a potential marker for the prevention and/or treatment of early DC.
Apoptosis of cardiomyocytes under a high-glucose environment contributes to the pathogenesis of DC (19), and inhibition of this pathophysiological process is considered to be a promising therapeutic target for DC (20). In the present study, it was illustrated that IncRNA NKILA overexpression and IncRNA NKILA knockdown accelerated and inhibited apoptotic cell death in cardiomyocytes under high-glucose treatment, respectively. Therefore, the suppression of lncRNA NKILA expression may inhibit the development of DC. Nevertheless, further clinical trial studies are required to confirmed the results of the present study. In addition, in the present study lncRNA NKILA expression did not change in the presence of high-glucose treatment in vitro. Therefore, lncRNA NKILA expression may be dysregulated during the formation of heart lesions in diabetic patients. 
Additionally, the present study showed that lncRNA NKILA levels were upregulated in patients with DC combined with other complications, including hand and food diseases, and retinopathy (data not shown). However, diabetic patients with multiple complications (except cardiomyopathy) exhibited no significant changes in lncRNA NKILA levels (data not shown), further implicating the specific involvement of IncRNA NKILA in DC.

A potential limitation in the data from the current study is that it did not elucidate the mechanism for the regulation of cardiomyocyte apoptosis by lncRNA NKILA. Preliminary studies have indicated that lncRNA NKILA has no binding partners with apoptosis mediators that are well-characterized in cardiomyocytes, including miR-133b-5 and miRNA-21 $(21,22)$. Therefore, the identification of the downstream effectors of IncRNA NKILA is needed.

In conclusion, the present study demonstrated that lncRNA NKILA was upregulated specifically in diabetic patients who developed DC, and lncRNA NKILA overexpression may contribute to the progression of DC by promoting cardiomyocyte apoptosis.

\section{Acknowledgements}

Not applicable.

\section{Funding}

No funding was received.

\section{Availability of data and materials}

The datasets used and/or analyzed during the present study are available from the corresponding author on reasonable request.

\section{Authors' contributions}

QL and SN designed the experiments. QL, PL, JS and SL performed the experiments. XY and YY assisted in performing the experiments and collected the data. SN drafted the manuscript. All authors read and approved the final version of the approved this manuscript.

\section{Ethics approval and consent to participate}

The present study was approved by the Ethics Committee of The People's Hospital of Puyang (Henan, China).

\section{Patient consent for publication}

Patients provided consent for the possible publication of this paper.

\section{Competing interests}

The authors declare that they have no competing interests.

\section{References}

1. Forbes JM and Cooper ME: Mechanisms of diabetic complications. Physiol Rev 93: 137-188, 2013.

2. Huang ES, Laiteerapong N, Liu JY, John PM, Moffet HH and Karter AJ: Rates of complications and mortality in older patients with diabetes mellitus: The diabetes and aging study. JAMA Intern Med 174: 251-258, 2014

3. Constantino MI, Molyneaux L, Limacher-Gisler F, Al-Saeed A, Luo C, Wu T, Twigg SM, Yue DK and Wong J: Long-term complications and mortality in young-onset diabetes: Type 2 diabetes is more hazardous and lethal than type 1 diabetes. Diabetes Care 36: 3863-3869, 2013.

4. Fuentes-Antrás J, Picatoste B, Ramírez E, Egido J, Tuñón J and Lorenzo Ó: Targeting metabolic disturbance in the diabetic heart. Cardiovasc Diabetol 14: 17, 2015.

5. Battiprolu PK, Lopez-Crisosto C, Wang ZV, Nemchenko A, Lavandero S and Hill JA: Diabetic cardiomyopathy and metabolic remodeling of the heart. Life Sci 92: 609-615, 2013.

6. Falcão-Pires I and Leite-Moreira AF: Diabetic cardiomyopathy: Understanding the molecular and cellular basis to progress in diagnosis and treatment. Heart Fail Rev 17: 325-344, 2012.

7. Huynh K, Bernardo BC, McMullen JR and Ritchie RH: Diabetic cardiomyopathy: Mechanisms and new treatment strategies targeting antioxidant signaling pathways. Pharmacol Ther 142: $375-415,2014$.

8. Jia G, DeMarco VG and Sowers JR: Insulin resistance and hyperinsulinaemia in diabetic cardiomyopathy. Nat Rev Endocrinol 12: 144-153, 2016.

9. Beltrami C, Angelini TG and Emanueli C: Noncoding RNAs in diabetes vascular complications. J Mol Cell Cardiol 89: 42-50, 2015.

10. Ponting CP, Oliver PL and Reik W: Evolution and functions of long noncoding RNAs. Cell 136: 629-641, 2009.

11. Zhuo C, Jiang R, Lin X and Shao M: LncRNA H19 inhibits autophagy by epigenetically silencing of DIRAS3 in diabetic cardiomyopathy. Oncotarget 8: 1429-1437, 2017.

12. Zhang M, Gu H, Chen J and Zhou X: Involvement of long noncoding RNA MALAT1 in the pathogenesis of diabetic cardiomyopathy. Int J Cardiol 202: 753-755, 2016.

13. Bian D, Gao C, Bao K and Song G: The long non-coding RNA NKILA inhibits the invasion-metastasis cascade of malignant melanoma via the regulation of NF- $\mathrm{BB}$. Am J Cancer Res 7: 28-40, 2017.

14. Bird L: IncRNA NKILA: A killer regulator. Nat Rev Immunol 18: 666-667, 2018

15. Livak KJ and Schmittgen TD: Analysis of relative gene expression data using real-time quantitative PCR and the 2(-Delta Delta C(T)) method. Methods 25: 402-408, 2001.

16. Tao F, Xu Y, Yang D, Tian B, Jia Y, Hou J and Dong M: LncRNA NKILA correlates with the malignant status and serves as a tumor-suppressive role in rectal cancer. J Cell Biochem 119: 9809-9816, 2018

17. Ke S, Li RC, Meng FK and Fang MH: NKILA inhibits NF- $\kappa B$ signaling and suppresses tumor metastasis. Aging (Albany NY) 10: 56-71,2018.

18. Jia G, Whaley-Connell A and Sowers JR: diabetic cardiomyopathy: A hyperglycaemia-and insulin-resistance-induced heart disease. Diabetologia 61: 21-28, 2018.

19. Wu W, Liu X and Han L: Apoptosis of cardiomyocytes in diabetic cardiomyopathy involves overexpression of glycogen synthase kinase-3 $\beta$. Biosci Rep 39: BSR20171307, 2019.

20. Diao X and Li G: Down-regulation of miR-30b reduces cardiomyocyte apoptosis by targeting $\mathrm{Bcl}-2$ in diabetic cardiomyopathy. Int J Clin Exp Med 10: 5296-5305, 2017.

21. Pan YL, Han ZY, He SF, Yang W, Cheng J, Zhang Y and Chen ZW: miR-133b-5p contributes to hypoxic preconditioning-mediated cardioprotection by inhibiting the activation of caspase- 8 and caspase-3 in cardiomyocytes. Mol Med Rep 17: 7097-7104, 2018

22. Shan ZX, Lin QX, Deng CY, Zhu JN, Mai LP, Liu JL, Fu YH, Liu XY, Li YX, Zhang YY, et al: miR-1/miR-206 regulate Hsp60 expression contributing to glucose-mediated apoptosis in cardiomyocytes. FEBS Lett 584: 3592-3600, 2010. 